May 2020

\title{
Research Publications in Web of Science shift from Bitcoin to Blockchain, with Focus on Computer Science, Engineering, Telecommunications, and Business Economics
}

\author{
Megan Price \\ San Jose State University, mmeprice@protonmail.com \\ Greta Snyder \\ San Jose State University, snyder.greta@gmail.com
}

Follow this and additional works at: https://scholarworks.sjsu.edu/ischoolsrj

Part of the Archival Science Commons, Cataloging and Metadata Commons, Collection Development and Management Commons, Information Literacy Commons, Museum Studies Commons, Scholarly Communication Commons, and the Scholarly Publishing Commons

\section{Acknowledgements}

Special thanks to Drs. Sandra Hirsh and Sue Alman, and Jason Griffey for their work on blockchain applications in the Library and Information Sciences

\section{Recommended Citation}

Price, M., \& Snyder, G. (2020). Research Publications in Web of Science shift from Bitcoin to Blockchain, with Focus on Computer Science, Engineering, Telecommunications, and Business Economics. School of Information Student Research Journal, 10(1). https://doi.org/10.31979/2575-2499.100111 Retrieved from https://scholarworks.sjsu.edu/ischoolsrj/vol10/iss1/11

This article is brought to you by the open access Journals at SJSU ScholarWorks. It has been accepted for inclusion in School of Information Student Research Journal by an authorized administrator of SJSU ScholarWorks. For more information, please contact scholarworks@sjsu.edu. 
Research Publications in Web of Science shift from Bitcoin to Blockchain, with Focus on Computer Science, Engineering, Telecommunications, and Business Economics

\section{Keywords}

blockchain, research, databases, technology, bitcoin

Acknowledgements

Special thanks to Drs. Sandra Hirsh and Sue Alman, and Jason Griffey for their work on blockchain applications in the Library and Information Sciences 
Research Publications in Web of Science shift from Bitcoin to Blockchain, with Focus on Computer Science, Engineering, Telecommunications, and Business Economics

A Review of:

M. Dabbagh, M. Sookhak, N. Sohrabi Safa (2019). The evolution of blockchain: A bibliometric study. IEEE Access, 7, 19212 - 19221. DOI:

10.1109/ACCESS.2019.2895646

Authors:

Abstract

Objective - To collect, characterize and analyze blockchain-related research publications found in the Web of Science database.

Design - Bibliometric study.

Setting - A science, social science, arts, and humanities citation index.

Subjects - Over 90 million records.

Methods - The authors developed five research questions to be answered in the study:

1. What is the distribution of Blockchain publications and citations over the recent years?

2. Which research areas have been mostly investigated in Blockchain based on the number of publications?

3. What are the most influential papers in Blockchain according to the number of citations?

4. What are the most popular publication venues for Blockchain papers?

5. What are the topmost supportive funding agencies of Blockchain papers?

To begin the process, the authors then selected Web of Science (WoS) as an appropriate search engine for beginning the search process, based on its reputation in the field, quality and quantity of publications, and search features. The authors identified and selected search vocabulary to include "Blockchain," "bitcoin," "cryptocurrency," "ethereum," and "smart contract." The search was conducted on a single day, after which the authors performed a descriptive analysis of the results as they related to the five initial questions.

Main Results - Findings show there has been a steep, close to $400 \%$, increase of blockchain-related papers published between 2013 and 2017, and a parallel increase in citations on WoS. Of the 25 research areas covered in WoS publications, the top four fields focusing on blockchain were Computer Science, 
Engineering, Telecommunications, and Business Economics, with Library and Information Science (LIS) as the ninth most researched. The citations also follow this pattern. The most influential paper, based on number of citations was "Blockchains and Smart Contracts for the Internet of Things," written by Konstantinos Christidis and Michael Devetsikiotis, and five of the top ten most cited papers were on research conducted in the USA. The most popular publications featuring articles on blockchain are Lecture Notes in Computer Science and IEEE Access. Finally, the study finds that the funding agencies most engaged with blockchain research are two research institutes in China, National Natural Science Foundation of China and National Key Research and Development Program of China, followed by the National Science Foundation in the USA.

Conclusion - While these findings identify current focus areas of blockchain research as related to economics and computer science, they also identify the wide range of other possible areas in the social sciences and humanities, including LIS. Notably, the LIS field has already begun its investigations into the use of Blockchain to facilitate library services such as interlibrary loan, and in archival practices such as certifying provenance.

The study identifies fields where Blockchain research has already begun to take hold, opening publication opportunities for authors and identifying which publications might yield the most citations for their own work, and assists authors, researchers and practicing professionals in identifying those organizations most willing to fund further blockchain research, exploration and implementation. Identification of key articles can provide authors with a starting point, when seeking key or influential works in their own fields to develop research, grant, and project proposals.

\section{Commentary}

The authors refer to the recent Miau and Yang (2018) bibliometric study, where Scopus was used as the database, and whose findings mimic the authors. The Miau and Yang (2018) study also found an increasing trend in blockchain research publication since 2016, and similarly identified the top ten papers published, selected and based on the number of publications on the subject. The authors also referred to the Zeng and $\mathrm{Ni}$ study (2018), which identified two additional databases, Ei Compendex and China National Knowledge Infrastructure, that produced different results. These studies, conducted using varied search engines and databases, provide future blockchain researchers with a wide array of data to support their work.

When the study is evaluated against Glynn's (2006) EBL Critical Appraisal Checklist criteria, it appears valid: Article inclusion and exclusion criteria are definitively outlined; the data collection methods were clearly articulated, and the results section effectively summarized potential applications 
and areas for further research. The methodology utilized was appropriate for the study and was stated in a way that would allow for replication, with two similar studies cited within the article. Finally, the outcomes of the study are clearly stated and discussed in relation to the data collection process.

To improve this study, the authors could more clearly indicate their reasons for decisions made during the study:

- How did the authors determine the initial 6 terms used in the query string?

- During data analysis, how did the authors determine which papers to include and exclude from the study?

- How, specifically, did this study build upon or align to the results found in the Miau and Yang and Zeng and Ni studies?

If the authors had provided a more detailed comparison table of their study's findings in relation to the other findings, this would enable future researchers to easily analyze and compare a greater amount of data.

This study may be useful for any LIS professional seeking funding and publication opportunities in blockchain-related research. The data provided by Dabbagh et al. offers a view of current research offerings and provides direction for applicable funding and publication sources. Dabbagah et al. also highlight the gap in research outside of the top four areas.

As the research trend continues to move away from a specific focus on Bitcoin, and toward its underlying technology, blockchain, this study catalyzes additional research opportunities and areas of real-world application. As the study's authors suggest, this work provides a stepping-stone for a future, even larger bibliometric study, that could identify additional trends and provide further insight into this quickly growing area.

\section{References}

Glynn, L. (2006). A critical appraisal tool for library and information research.

Library Hi Tech, 24(3), 387-399. https://doi.org/10.1108/07378830610692154

Miau, S., \& Yang, J.-M. (2018). Bibliometrics-based evaluation of the Blockchain research trend: 2008. March 2017. Technology Analysis \& Strategic Management, 30(9), 1029-1045. https://doi.org/10.1080/09537325.2018.1434138

Zeng, S., Ni, X., Yuan, Y., \& Wang, F.-Y. (2018). A bibliometric analysis of Blockchain research. In 2018. IEEE Intelligent Vehicles Symposium (IV), 102-107. https://doi.org/10.1109/IVS.2018.8500606 